\title{
Real principal value integrals *
}

\author{
Ph.Jacobs
}

\begin{abstract}
We study principal value integrals of multi-valued differential forms on compact spaces, as introduced by Langlands. Using resolutions of singularities we extend Langlands definition to the case in which the differential form may have no normal crossings. We show by an example that for non-compact spaces the principal value integral associated to a compactification may depend on the compactification.

Principal value integrals appear as residues of poles of distributions $|f|^{\lambda}$ and as coefficients of asymptotic expansions of oscillating integrals and fibre integrals.
\end{abstract}

\section{Introduction}

In this paper we study real principal value integrals. Real principal value integrals and their non-Archimedean analogue were first introduced by Langlands in view of the Langlands program [L1, L2, LS1, LS2]. We should note that this notion of principal value integral differs from the more classical notion of Cauchy [Ho, p.73]. In this introduction we first give a short overview of the paper, afterwards we briefly explain the connection between coefficients of asymptotic expansions of oscillating integrals and residues of poles of distributions $|f|^{\lambda}$, see [A-V-G, II,$\S 7]$, [Den2, I1, I2, Lae] for more details. In some interesting cases both can be expressed in terms of principal value integrals [J2].

On a real-analytic manifold $X$ we take a meromorphic differential form $\omega$ of higher degree $d$ (see section 2) and a real-valued $C^{\infty}$-function $\rho$. To this data we associate the principal value integral of $\rho\left|\omega^{1 / d}\right|$ on $X$, denoted by $P V \int_{X} \rho\left|\omega^{1 / d}\right|$. However to obtain a well-defined notion we need two conditions to be satisfied

1. $\omega$ has normal crossings on $\operatorname{supp}(\rho)$, and

*key words: principal values, singularities, meromorphic continuations MSC (1991): 26E05, 32S05, 32S45 
2. $\omega^{1 / d}$ has no integral poles on $\operatorname{supp}(\rho)$.

These conditions are explained in section 4. First we define principal value integrals on (suitable) charts on $X$. Then we define principal value integrals on the manifold $X$ by choosing a covering of $X$ with (suitable) charts and a $C^{\infty}$-partition of unity with respect to this covering and glueing the principal value integrals on the charts. Of course one has to check that this definition is independent of the chosen covering and partition of unity. This is carefully done in sections 3 to 5 . (In [L2] Langlands doesn't check this independency (since he doesn't need it) although he remarks "It is best of course to verify that the principal values introduced here are well-defined".)

In section 7 we generalize the definition of principal value integrals, considering also the case in which $\omega$ doesn't have normal crossings on $\operatorname{supp}(\rho)$, i.e. omitting condition 1. In this case we choose a (suitable) resolution $\pi: Y \rightarrow X$ such that $\pi^{*} \omega$ has normal crossings on $\operatorname{supp}\left(\pi^{*} \omega\right)$. If moreover $\left(\pi^{*} \omega\right)^{1 / d}$ has no integral poles on $\operatorname{supp}\left(\pi^{*} \rho\right)$ then we define

$$
P V \int_{X} \rho\left|\omega^{1 / d}\right|:=P V \int_{Y} \pi^{*}(\rho)\left|\left(\pi^{*} \omega\right)^{1 / d}\right| .
$$

We check that this definition is independent of the chosen (suitable) resolution.

If the space $X$ is compact, then one can take $\rho=1$ and one has a welldefined notion of the principal value integral of $\left|\omega^{1 / d}\right|$ on $X$. One can try to generalize this for non-compact spaces $X$ as follows. Suppose that $i: X \rightarrow Y$ is a compactification of $X$ such that $i_{*} \omega$, the extension of $\omega$ to $Y$, satisfies conditions 1 and 2. Then we could try to define $P V \int_{X}\left|\omega^{1 / d}\right|$ as $P V \int_{Y}\left|\left(i_{*} \omega\right)^{1 / d}\right|$. However we show in section 8 , by an example, that the result may depend on the chosen compactification.

In section 9 we prove a proposition about limits of principal value integrals. An application of this proposition can be found in [J, Remark 8.2.7].

Now we discuss the connection between distributions $|f|^{s}$, oscillating integrals and principal value integrals. Let $f: \mathbb{R}^{m} \rightarrow \mathbb{R}$ be a non-constant real-analytic function with only isolated singularities. Let $\varphi: \mathbb{R}^{m} \rightarrow \mathbb{R}$ be a $C^{\infty}$-function with compact support. Then one considers the integral

$$
I(\tau):=\int_{\mathbb{R}^{m}} e^{i \tau f(x)} \varphi(x) d x,
$$

where $\tau$ is a real parameter, $x=\left(x_{1}, \ldots, x_{m}\right)$ and $d x=d x_{1} \wedge \cdots \wedge d x_{m}$. The function $I(\tau)$ has an asymptotic expansion for $\tau \rightarrow+\infty$

$$
I(\tau) \approx \sum_{\alpha} \sum_{k=0}^{m-1} a_{k, \alpha}(\varphi) \tau^{\alpha}(\ln \tau)^{k}
$$


where the coefficients $a_{k, \alpha}$ are distributions of $\varphi$ and where $\alpha$ runs through a finite set $A$ of numbers in descending arithmetic progressions. One can write down these arithmetic progressions in terms of the numerical data $\left(N_{i}, \nu_{i}\right), i \in I$, of an embedded resolution $\pi: Y \rightarrow \mathbb{R}^{m}$ of the singularities of $f$. The set $A$ consists of the numbers in the aritmethic progressions

$$
\frac{-\nu_{i}}{N_{i}}, \frac{-\left(\nu_{i}+1\right)}{N_{i}}, \frac{-\left(\nu_{i}+2\right)}{N_{i}}, \ldots \text { for } i \in I .
$$

Now let $\lambda$ be a complex parameter with real part $\Re(\lambda)>>0$ and define the functions

$$
G_{ \pm}(\lambda):=\int_{f>0} f^{\lambda} \varphi d x \pm \int_{f<0}(-f)^{\lambda} \varphi d x .
$$

Then

$$
\begin{gathered}
G_{+}(\lambda)=\int_{\mathbb{R}^{m}}|f|^{\lambda} \varphi d x \text { and } \\
G_{-}(\lambda)=\int_{\mathbb{R}^{m}} \operatorname{sgn}(f)|f|^{\lambda} \varphi d x,
\end{gathered}
$$

where sgn denotes the sign function. So we obtain the classical distribution $|f|^{\lambda}$ and it's twisted version $\operatorname{sgn}(f)|f|^{\lambda}$ (twisted by the character sgn). One can show that these functions have meromorphic extensions to the complex plane with poles $\alpha$ in the set $A$, therefore called the set of candidate poles. The coefficient $a_{k, \alpha}(\varphi)$ in the asymptotic expansion of $I(\tau)$ can easily be expressed in terms of the coefficients $b_{l, \alpha}^{ \pm}(\varphi)$ of $(\lambda-\alpha)^{-(l+1)}, l \geq k$, in the Laurent expansions of $G_{ \pm}(\lambda)$.

Let $\alpha \in A$ be a candidate pole. Using the resolution $\pi$ one finds a nonnegative integer $k_{\alpha}$ such that $b_{k, \alpha}^{ \pm}(\varphi)=0$ for all $\varphi$ and all $k>k_{\alpha}$. Then, for most $\varphi, k_{\alpha}$ is the expected order of $\alpha$ as pole of $G_{ \pm}(\lambda)$. Let $\beta$ be the maximum of $A$ such that $a_{k_{\beta}, \beta}(\varphi) \tau^{\beta}(\ln \tau)^{k_{\beta}}$ is the dominating term in the asymptotic expansion of $I(\tau)$. In $[\mathrm{J} 2]$ we show that $b_{k_{\beta}, \beta}^{ \pm}(\varphi)$ and hence $a_{k_{\beta}, \beta}(\varphi)$ can be expressed in terms of real principal value integrals (of a meromorphic differential form of higher degree).

In [D-J] we state a theorem that gives a condition for the vanishing of principal value integrals in terms of cohomology. Hence, in some cases, one can use this theorem to prove that $a_{k_{\beta}, \beta}(\varphi)=0$. We will now formulate this theorem.

Let $X$ be a non-singular complex projective algebraic variety defined over $\mathbb{R}$ of complex dimension $m$ such that $X(\mathbb{R}) \neq \emptyset$. Let $\omega$ be a rational differential $m$-form of degree $d$ on $X$ defined over $\mathbb{R}$. (This is the algebraic analogue of a meromorphic differential form of degree $d$.) Let $|\operatorname{div}(\omega)|$ denote the support of the divisor of $\omega$. Let $\chi: X(\mathbb{R}) \rightarrow\{1,-1\}$ be a function that is constant on the 
connected components of $X(\mathbb{R})-|\operatorname{div}(\omega)|$. We assume that $|\operatorname{div}(\omega)|$ has normal crossings over $\mathbb{R}$, meaning that it has normal crossings and that each irreducible component containing an $\mathbb{R}$-rational point is defined over $\mathbb{R}$. Moreover we assume that $\omega^{1 / d}$ has no integral poles on $X$. Then we can define $P V \int_{X(\mathbb{R})} \chi\left|\omega^{1 / d}\right|$, the (twisted) principal value integral of $\chi\left|\omega^{1 / d}\right|$ over $X(\mathbb{R})$, as above. Let $\mathcal{L}\left(\omega^{1 / d}\right)$ be the locally constant sheaf of $\mathbb{C}$-vectorspaces on $X-|\operatorname{div}(\omega)|$ associated to $\omega^{1 / d}$, which is locally free of rank 1 . A non-zero section of $\mathcal{L}\left(\omega^{1 / d}\right)$ on a connected open $U$ is an analytic branch of $\omega^{1 / d}$ on $U$ multiplied with a complex number.

( 1.1 ) Theorem. If $H^{m}\left(X(\mathbb{C})-|\operatorname{div}(\omega)|, \mathcal{L}\left(\omega^{1 / d}\right)\right)=0$ then

$$
P V \int_{X(\mathbb{R})} \chi\left|\omega^{1 / d}\right|=0 .
$$

For a nice overview of the proof we refer to [D-J]. The whole proof can be found in $[\mathrm{J}]$.

Finally I'd like to thank Prof. Jan Denef for introducing me to the subject and for the many interesting discussions we had about this topic.

\section{Real meromorphic differential forms of higher degree}

( 2.1 ) In this section we define meromorphic forms of higher degree and we state some lemmas about it. The proofs of the lemmas are easy and left to the reader. They are based on the following well-known facts: the local rings of a real-analytic manifold $X$ are UFD's (unique factorisation domains) and relatively prime elements in the local ring of a point of $X$ stay relatively prime in a small enough neighborhood of the point.

( 2.2 ) Data. Let $X$ be a real-analytic manifold of dimension $m$. Let $\mathcal{O}$ denote the sheaf of real-analytic functions on $X$. Let $\Omega^{m}$ denote the sheaf of realanalytic differential $m$-forms on $X$. Let $d$ be a positive integer and $\left(\Omega^{m}\right)^{\otimes d}$ the $d$-th tensor power of $\Omega^{m}$ over the sheaf $\mathcal{O}$, i.e. $\left(\Omega^{m}\right)^{\otimes d}=\Omega^{m} \otimes_{\mathcal{O}} \Omega^{m} \otimes_{\mathcal{O}} \cdots \otimes_{\mathcal{O}} \Omega^{m}$ ( $d$ times).

( 2.3 ) Definition. If $U$ is an open subset of $X$ and $\omega$ belongs to $\left(\Omega^{m}\right)^{\otimes d}(U)$, then we say that $\omega$ is a real-analytic differential $m$-form of degree $d$ on $U$.

( 2.4 ) Remark. Let $x: U \rightarrow \mathbb{R}^{m}$ be a chart on $X$. We denote the $i$-th coordinate function with respect to $x$ by $x_{i}$, i.e. $x_{i}=p_{i} \circ x$ where $p_{i}: \mathbb{R}^{m} \rightarrow \mathbb{R}$ 
is the projection to the $i$-th coordinate. We also write $d x$ for $d x_{1} \wedge \cdots \wedge d x_{m}$ and $(d x)^{d}$ for $d x \otimes \cdots \otimes d x$ ( $d$ times). Let $\omega$ be a real-analytic differential $m$-form of degree $d$ on $U$, then there exists a real-analytic function $f$ on $U$ such that $\omega=f(d x)^{d}$. If we choose another chart $y: V \rightarrow \mathbb{R}^{m}$ on $X$ such that $U$ meets $V$ then $\omega=f \operatorname{det}\left(\frac{\partial x}{\partial y}\right)^{d}(d y)^{d}$ on $U \cap V$.

( 2.5 ) Definition. A pair $(P, \omega)$ is called a real-meromorphic differential $m$-form of degree $d$ on an open subset $V$ of $X$ if

1. $P$ is a closed subset of $V$,

2. $\omega$ is a real-analytic differential $m$-form of degree $d$ on $V-P$,

3. for each point $p$ in $V$ there exists a chart $x: U \rightarrow \mathbb{R}^{m}$ on $V$ and realanalytic functions $g, h$ on $U$ such that

(a) $U$ is a connected open neighborhood of $p$ in $V$ and $h$ is not identically zero on $U$,

(b) $P \cap U=\{q \in U \mid h(q)=0\}$,

(c) $\omega=\frac{g}{h}(d x)^{d}$ on $U-P$ and

(d) the germs of $g$ and $h$ in the local ring $\mathcal{O}_{X, p}$ are relatively prime.

The set $P$ is called the polar locus of $(P, \omega)$.

( 2.6 ) Remark. It follows from the definition that $P$ is a real-analytic subset of $V$ of codimension at least 1 .

( 2.7 ) Remark. If we take a chart $x: U \rightarrow \mathbb{R}^{m}$ as in definition (2.5) then one easily sees by changing coordinates that any chart $y: U \rightarrow \mathbb{R}^{m}$ satisfies condition 3.

( 2.8$)$ Lemma. Let $\left(P_{1}, \omega_{1}\right)$ and $\left(P_{2}, \omega_{2}\right)$ be two real-meromorphic differential $m$-forms of degree $d$ on $X$ that coincide on $X-\left(P_{1} \cup P_{2}\right)$. Then $\left(P_{1}, \omega_{1}\right)=\left(P_{2}, \omega_{2}\right)$.

( 2.9 ) Lemma. Let $A$ be a closed subset of $X$ such that $X-A$ is dense in $X$. Let $\omega$ be a real-analytic differential $m$-form of degree $d$ on $X-A$. Suppose that for each point $p$ in $A$ there exists a chart $x: U \rightarrow \mathbb{R}^{m}$ and real-analytic functions $g, h$ on $U$ such that

1. $U$ is a path-connected open neighborhood of $p$ and $h$ is not identically zero on $U$, 


$$
\begin{aligned}
& \text { 2. } A \cap U=\{q \in U \mid h(q)=0\} \text { and } \\
& \text { 3. } \omega=\frac{g}{h}(d x)^{d} \text { on } U-A \text {. }
\end{aligned}
$$

Then there exists a closed subset $P$ of $A$ and a real-analytic differential $m$-form $\hat{\omega}$ of degree $d$ on $X-P$ such that $(P, \hat{\omega})$ is a real-meromorphic differential $m$ form of degree $d$ on $X$ and $\hat{\omega}$ restricts to $\omega$ on $X-A$. Moreover the pair $(P, \hat{\omega})$ is uniquely determined by these conditions.

( 2.10 ) Definition. The real-meromorphic differential $m$-form $(P, \hat{\omega})$ of degree $d$ on $X$ of the previous lemma is called the meromorphic extension of $\omega$ to $X$.

( 2.11 ) Convention. Sometimes we will say: "Let $\omega$ be a real-meromorphic differential $m$-form of degree $d$ on $X$." Then we will mean that $\omega$ is a realanalytic differential $m$-form of degree $d$ on $X-P$ for some closed subset $P$ of $X$, such that $(P, \omega)$ is a real-meromorphic differential $m$-form of degree $d$ on $X$.

( 2.12 ) Definition. Let $g, h$ be real-analytic functions on an open subspace $U$ of $X$. We say that $g$ and $h$ are locally relatively prime on $U$ if the germs of $g$ and $h$ are relatively prime in each local ring $\mathcal{O}_{X, p}$ with $p$ in $U$.

( 2.13 ) Lemma. Let $\omega$ be a real-meromorphic differential $m$-form of degree $d$ on $X$. Then for each point $p$ in $X$ there exists a chart $x: U \rightarrow \mathbb{R}^{m}$ with $p$ in $U$ and locally relatively prime real-analytic functions $g, h$ on $U$ such that $\omega=\frac{g}{h}(d x)^{d}$ on $U$. The functions $g$ and $h$ are uniquely determined up to multiplication with nowhere vanishing real-analytic functions on $U$.

( 2.14 ) Definition. Let $\omega$ be a real-meromorphic differential $m$-form of degree $d$ on $X$. We define the zero locus of $\omega$ as the set of points $p$ in $X$ for which there exists a chart $x: U \rightarrow \mathbb{R}^{m}$ on $X$ and locally relatively prime real-analytic functions $g, h$ on $U$ such that $p$ lies in $U, \omega=\frac{g}{h}(d x)^{d}$ on $U$ and $g(p)=0$. We define the locus of $\omega$ as the union of its zero locus and its polar locus.

\section{Meromorphic continuations of integrals}

In this section we state a well-known lemma on meromorphic continuations of integrals. Similar results in the complex case can be found in [Ba1, Ba4]. 
( 3.1 ) Data. Let $m, l \in \mathbb{N}, m \neq 0$. Let $U$ be open in $\mathbb{R}^{m}$. Let $\varphi: U \rightarrow \mathbb{R}$ be a $C^{\infty}$-function with compact support in $U$. Let $\eta=\left(\eta_{i}\right)$ be a $l$-tuple of real analytic functions $\eta_{i}: U \rightarrow \mathbb{R}$ non-vanishing on $U$.

( 3.2 ) Notation. Denote $|\eta|^{t}=\prod_{i=1}^{l}\left|\eta_{i}\right|^{t_{i}},|x|^{s}=\prod_{i=1}^{m}\left|x_{i}\right|^{s_{i}}$ for $t \in \mathbb{C}^{l}$, $s \in \mathbb{C}^{m}$ and $d x=d x_{1} \wedge d x_{2} \wedge \cdots \wedge d x_{m}$. Let $g n$ denote the sign function, i.e. $g n(x)=1$ for $x \in \mathbb{R}, x \geq 0$ and $g n(x)=-1$ for $x \in \mathbb{R}, x<0$. Let $\operatorname{sgn}(x)^{k}:=\prod_{i=1}^{m} \operatorname{sgn}\left(x_{i}\right)^{k_{i}}$ and $\frac{\partial^{k}}{\partial x^{k}}:=\prod_{i=1}^{m} \frac{\partial^{k_{i}}}{\partial x_{i} k_{i}}$ for $k \in \mathbb{N}^{m}$. Let $V$ be a nonempty open subset of $\mathbb{C}^{r}$ and $f$ an analytic function from $V$ to $\mathbb{C}$. If $f$ has a (unique) meromorphic continuation to $\mathbb{C}^{r}$ then we denote it by $[f]_{m c}$. Let $f: \mathbb{C}^{r} \rightarrow \mathbb{C}$ be a meromorphic function which is holomorphic in a point $\alpha \in \mathbb{C}^{r}$. Then we denote the value of $f$ in $\alpha$ by $[f(s)]_{s=\alpha}$.

(3.3 ) Lemma. Assume the data (3.1) and notation (3.2). Fix $r \in \mathbb{N}^{m}$. Then

1. $\int_{U} \operatorname{sgn}(x)^{r} \varphi|\eta|^{t}|x|^{s} d x$ is an analytic function on $\Re\left(s_{i}\right)>-1$.

2. $\int_{U} \operatorname{sgn}(x)^{r} \varphi|\eta|^{t}|x|^{s} d x$ has a unique meromorphic continuation to $\mathbb{C}^{l+m}$, denoted by $\left[\int_{U} \operatorname{sgn}(x)^{r} \varphi|\eta|^{t}|x|^{s} d x\right]_{m c}$, with polar locus contained in the set $P=\left\{(t, s) \in \mathbb{C}^{l+m} \mid \exists i: s_{i} \in \mathbb{Z}-\mathbb{N}\right\}$.

3. Let $k \in \mathbb{N}^{m}$ then on $\Re\left(s_{i}\right)>-1-k_{i}$

$$
\left[\int_{U} \operatorname{sgn}(x)^{r} \varphi|\eta|^{t}|x|^{s} d x\right]_{m c}=\left(\prod_{i=1}^{m} \prod_{j_{i}=1}^{k_{i}} \frac{-1}{s_{i}+j_{i}}\right) \int \operatorname{sgn}(x)^{r+k} \frac{\partial^{k}}{\partial x^{k}}\left(\varphi|\eta|^{t}\right)|x|^{s+k} d x
$$

4. Let $(\epsilon, \alpha) \in \mathbb{C}^{l+m}-P$. Let $I$ be a subset of $\{1, \ldots, m\}$ containing all indices $i$ for which $\operatorname{Re}\left(\alpha_{i}\right) \leq-1$. Let $J:=\{1, \ldots, m\}-I$. Let $s_{I}:=\left(s_{i}\right)_{i \in I}$, $\alpha_{I}:=\left(\alpha_{i}\right)_{i \in I}, s_{J}:=\left(s_{i}\right)_{i \in J}$ and $\alpha_{J}:=\left(\alpha_{i}\right)_{i \in J}$. Then

$$
\begin{gathered}
{\left[\left[\int_{U} \operatorname{sgn}(x)^{r} \varphi|\eta|^{t}|x|^{s} d x\right]_{m c}\right]_{(t, s)=(\epsilon, \alpha)}} \\
=\left[\left[\int_{U} \operatorname{sgn}(x)^{r} \varphi\left[|\eta|^{t}\right]_{t=\epsilon}|x|^{s} d x\right]_{m c}\right]_{s=\alpha} \\
=\left[\left[\int_{U} \operatorname{sgn}(x)^{r} \varphi|\eta|^{t}\left[|x|^{s}\right]_{s_{J}=\alpha_{J}} d x\right]_{m c}\right]_{\left(t, s_{I}\right)=\left(\epsilon, \alpha_{I}\right)} \\
=\left[\left[\int_{U} \operatorname{sgn}(x)^{r} \varphi\left[|\eta|^{t}|x|^{s}\right]_{\left(t, s_{J}\right)=\left(\epsilon, \alpha_{J}\right)} d x\right]_{m c}\right]_{s_{I}=\alpha_{I}} .
\end{gathered}
$$




\section{Principal value integrals on charts}

( 4.1 ) Data. Let $X$ be a $m$-dimensional real-analytic manifold. Let $\omega$ be a real meromorphic differential $m$-form of degree $d$ on $X$. Let $\rho$ be a $C^{\infty}$-function $X \rightarrow \mathbb{R}$. Denote the support of $\rho$ by $\operatorname{supp}(\rho)$.

\section{( 4.2 ) Definition.}

1. We say that the chart $x: U \rightarrow \mathbb{R}^{m}$ on $X$ is an admissible chart for $(X, \omega)$ with data $(\xi ; \beta)$ if $\omega=\xi x^{\beta}(d x)^{d}$ on $U$, where $\xi$ is a nowhere vanishing real-analytic function on $U$ and $\beta \in \mathbb{Z}^{m}$.

2. We say that $\omega$ has normal crossings in a point $p$ of $X$ if there exists an admissible chart $x: U \rightarrow \mathbb{R}^{m}$ for $(X, \omega)$, centered in $p$ (i.e. $x(p)=0$ ). We say that $\omega$ has normal crossings in a subset $K$ of $X$ if it has normal crossings in every point of $K$.

3. We say that a chart $x: U \rightarrow \mathbb{R}^{m}$ on $X$ is a pv-chart for $(X, \omega)$ if $x$ is an admissible chart for $(X, \omega)$ with data $(\xi ; \beta)$ where the $\beta_{i} / d \notin \mathbb{Z}-\mathbb{N}$. We say that $x$ is a pv-chart for $(X, \omega, \rho)$ if $x$ is a pv-chart for $(X, \omega)$ and $\rho$ has compact support in $U$ (i.e. $\operatorname{supp}(\rho)$ is compact and it is contained in $U$ ).

4. Suppose that $\omega$ has normal crossings in a point $p$ of $X$. We say that $\omega^{1 / d}$ has no integral poles in $p$ if there exists a pv-chart $x: U \rightarrow \mathbb{R}^{m}$ for $(X, \omega)$ centered in $p$. Suppose that $\omega$ has normal crossings on a subset $K$ of $X$. We say that $\omega^{1 / d}$ has no integral poles on $K$ if it has no integral poles in every point of $K$.

( 4.3 ) Definition. If $x: U \rightarrow \mathbb{R}^{m}$ is a pv-chart for $(X, \omega, \rho)$ with data $(\xi ; \beta)$ then by lemma (3.3) the integral $\int_{U} \rho|\xi|^{1 / d}|x|^{\beta / d+s}|d x|$ exists for $\operatorname{Re}(s)>>0$ and has a (unique) meromorphic continuation to $\mathbb{C}^{m}$ (denoted by the square brackets and the index $m c$ ) that is holomorphic in $s=0$. (Here $s$ is a onedimensional complex variable.) Then we define

$$
\underset{x}{P V} \int \rho\left|\omega^{1 / d}\right|:=\left[\left[\int_{U} \rho|\xi|^{1 / d}|x|^{\beta / d+s}|d x|\right]_{m c}\right]_{s=0},
$$

called the principal value integral of $\rho\left|\omega^{1 / d}\right|$ on $x$.

( 4.4 ) Remark. Let $x: U \rightarrow \mathbb{R}^{m}$ be a pv-chart for $(X, \omega, \rho)$ and $W$ an open subset of $U$ containing $\operatorname{supp}(\rho)$. Then $x \mid W$ is a pv-chart for $(X, \omega, \rho)$ and $P \underset{x}{P V} \int \rho\left|\omega^{1 / d}\right|=\underset{x \mid W}{P V} \int \rho\left|\omega^{1 / d}\right|$. 
( 4.5 ) Lemma. Let $x: U \rightarrow \mathbb{R}^{m}$ be a pv-chart for $(X, \omega, \rho)$ with data $(\xi, \beta)$. Let $V$ be an open neighborhood of a point $q$ such that $\operatorname{supp}(\rho) \subset V \subset U$. Suppose that for all $i$ with $\beta_{i} \neq 0$ the zero locus of $x_{i}$ meets $V$ if and only if it contains q. Let $y: V \rightarrow \mathbb{R}^{m}$ be the translated chart defined by $y_{i}(r):=x_{i}(r)-x_{i}(q)$ for all $i, 1 \leq i \leq m$, and for all $r$ in $V$. Define $\gamma_{i}:=\beta_{i}$ if $\beta_{i} \neq 0$ and $\gamma_{i}:=0$ otherwise. Define $\psi:=\xi \prod_{i \mid x_{i}(q) \neq 0} x_{i}{ }^{\beta_{i}}$.

Then $y$ is a pv-chart for $(X, \omega, \rho)$, centered in $q$, with data $(\psi ; \gamma)$. Moreover

$$
\underset{x}{P V} \int \rho\left|\omega^{1 / d}\right|=\underset{y}{P V} \int \rho\left|\omega^{1 / d}\right|
$$

Proof: This follows from lemma (3.3).

( 4.6 ) Lemma. Let $x: U \rightarrow \mathbb{R}^{m}$ and $y: V \rightarrow \mathbb{R}^{m}$ be two admissible charts for $(X, \omega)$. Suppose that $y$ is centered in a point $q$ of $U \cap V$ and has data $(\xi ; \beta)$. Then there exists an open neighborhood $W$ of $q$ in $U \cap V$ and a permutation $\sigma$ of $\{1, \ldots, m\}$ such that for each $i$ with $\beta_{i} \neq 0$ there exists a real-analytic nowhere vanishing function $f_{i}$ on $W$ such that $x_{\sigma(i)}=f_{i} y_{i}$ on $W$.

Proof: Let $D_{1}, D_{2}, \ldots, D_{t}$ be the irreducible components of $D \cap U \cap V$ going through $q$, where $D$ denotes the locus of $\omega$. After a permutation of the $x_{i}$ and a permutation of the $y_{i}$ we may assume that there is a neighborhood $N$ of $q$ in $U \cap V$ such that $t \leq m, \beta_{i} \neq 0$ if and only if $1 \leq i \leq t$, and $D_{i}=\left\{p \in N \mid x_{i}(p)=0\right\}=\left\{p \in N \mid y_{i}(p)=0\right\}$. There exists an open neighborhood $V_{y}$ of $q$ in $N$ on which we can express the $x_{i}$ as convergent power series in $y$. Thus, by the description of $D_{i}$, there exists a convergent power series $f_{i}$ in $y$ on $V_{y}$ such that $x_{i}=f_{i} y_{i}$ on $V_{y}$ for $1 \leq i \leq t$. But the $x_{i}, 1 \leq i \leq t$, can be extended to a system of local coordinates on $X$ in a neighborhood of $q$ since $y$ is centered in $q$. Hence there is also an open neighborhood $V_{x}$ of $q$ in $N$ and real-analytic functions $g_{i}$ on $V_{x}$, for $1 \leq i \leq t$, such that $y_{i}=g_{i} x_{i}$ on $V_{x}$. But then $x_{i}=f_{i} g_{i} x_{i}$ on $V_{x} \cap V_{y}$ for $1 \leq i \leq t$. But since $\mathcal{O}_{X, q}$ is a domain we then have $f_{i} g_{i}=1$ in the local ring $\mathcal{O}_{X, q}$. Hence there exists an open neighborhood $W$ of $q$ in $V_{x} \cap V_{y}$ such that $f_{i} g_{i}=1$ on $W$ for all $i$ with $1 \leq i \leq t$. This proves the proposition.

\section{Principal value integrals on manifolds}

( 5.1 ) Data. Let $X$ be a $m$-dimensional real-analytic manifold. Let $\omega$ be a real meromorphic differential $m$-form of degree $d$ on $X$. Let $\rho: X \rightarrow \mathbb{R}$ be a 
$C^{\infty}$-function with compact support.

( 5.2 ) Assumption. In this section we assume that $\omega^{1 / d}$ has no integral poles in $\operatorname{supp}(\rho)$ (see definition (4.2)).

( 5.3 ) Definition. Let $\mathcal{U}=\left\{U_{\alpha} \mid \alpha \in A\right\}$ be an open covering of a subset $K$ of a topological space $X$ (i.e. $K \subset \underset{\alpha \in A}{\cup} U_{\alpha}$ ). Then a compact partition of unity with respect to the covering $\mathcal{U}$ of $K$ is a collection of continuous maps $\left\{f_{\beta}: X \rightarrow[0,1] \mid \beta \in B\right\}$ such that

1. there is a locally finite open refinement $\mathcal{V}=\left\{V_{\beta} \mid \beta \in B\right\}$ of $\mathcal{U}$ such that $\operatorname{supp}\left(f_{\beta}\right)$ is a compact subset of $V_{\beta}$ for all $\beta \in B$, and

2. $\sum_{\beta} f_{\beta}(x)=1$ for all $x \in K$.

If $X$ is a $C^{\infty}$-manifold the compact partition of unity is said to be $C^{\infty}$ if the functions $f_{\beta}$ are $C^{\infty}$.

( 5.4 ) Theorem. Let $X$ be a locally compact, Hausdorff, paracompact topological space and $\mathcal{U}=\left\{U_{\alpha} \mid \alpha \in A\right\}$ an open covering of a compact subset $K$ of $X$. Then there exists a finite compact partition of unity $\left\{f_{\beta} \mid \beta \in B\right\}$ with respect to the covering $\mathcal{U}$ of $K$. If $\mathcal{U}$ is finite then we can take $B=A$ and $\operatorname{supp}\left(f_{\alpha}\right) \subset U_{\alpha}$ for each $\alpha$ in $A$. If $X$ is a $C^{\infty}$-manifold the compact partition of unity can be taken $C^{\infty}$.

( 5.5 ) Convention. Let $\left\{\varphi_{p}\right\}_{p \in P}$ be a compact partition of unity with respect to a covering $\left\{U_{p}\right\}_{p \in P}$ of a subset $K$ of $X$ (see definition (5.3)). Then in the rest of this chapter we will always assume that $\operatorname{supp}\left(\varphi_{p}\right) \subset U_{p}$.

( 5.6 ) Lemma. There exists a finite set of triples $\left\{\left(U_{p}, \varphi_{p}, x_{p}\right)\right\}_{p \in P}$ such that $\left\{U_{p}\right\}_{p \in P}$ is a covering of $\operatorname{supp}(\rho),\left\{\varphi_{p}\right\}_{p \in P}$ is a $C^{\infty}$ compact partition of unity with respect to this covering and $x_{p}: U_{p} \rightarrow \mathbb{R}^{m}$ is a pv-chart for $\left(X, \omega, \rho \varphi_{p}\right)$ for all $p \in P$.

Proof: This follows directly from the assumption (5.2).

( 5.7 ) Lemma. Let $x$ be a pv-chart for $(X, \omega, \rho)$. Let $\left\{U_{p}\right\}_{p \in P}$ be a finite open covering of supp $(\rho)$ in $X$. Let $\left\{\varphi_{r}\right\}_{r \in R}$ be a compact partition of unity with respect to this covering. Then

$$
\underset{x}{P V} \int \rho\left|\omega^{1 / d}\right|=\sum_{r \in R} P_{x} \int \rho \varphi_{r}\left|\omega^{1 / d}\right|
$$


Proof: This follows easily from the definition of pv-integrals and the fact that integrals, meromorphic continuation and evaluation commute with finite sums.

( 5.8 ) Lemma. Let $x$ : $U_{x} \rightarrow \mathbb{R}^{m}$ and let $y: U_{y} \rightarrow \mathbb{R}^{m}$ be two pv-charts for $(X, \omega, \rho)$ with $y$ centered in a point $p \in U_{x} \cap U_{y}$. Then

$$
\underset{x}{P V} \int \rho\left|\omega^{1 / d}\right|=\underset{y}{P V} \int \rho\left|\omega^{1 / d}\right| .
$$

Proof: Let $x$ have data $(\xi, \gamma)$ and define the set $I_{x}$ as the set containing all indices $i$ with $\gamma_{i} \neq 0$. Let $I_{y}$ be similarly defined. By lemma (5.7) and lemma (4.5) we may suppose that $U_{y}$ is like the set $W$ in proposition (4.6). By remark (4.4) we may even assume that $U_{y} \subset U_{x}$. Thus after renumbering the $y_{i}$ we have $I_{y}$ is a subset of $I_{x}$ and there exist nowhere vanishing real-analytic functions $f_{i}$ on $U_{y}$ for all $i$ in $I_{x}$ such that on $U_{y}$ we have $x_{i}=f_{i} y_{i}$ for $i \in I_{y}$ and $x_{i}=f_{i}$ for $i \in I_{x}-I_{y}$. There also exists a nowhere vanishing real-analytic function $\zeta$ on $U_{y}$ such that $d x=\zeta d y$ on $U_{y}$. Then

$$
\omega=\xi \zeta^{d} \prod_{i \in I_{x}} f_{i}^{\gamma_{i}} \prod_{i \in I_{y}} y_{i}^{\gamma_{i}}(d y)^{d} \text { on } U_{y} .
$$

Denote $\alpha_{i}:=\gamma_{i} / d, s_{x}:=\left(s_{i}\right)_{i \in I_{x}}$ and $s_{y}:=\left(s_{i}\right)_{i \in I_{y}}$. Then

$$
\begin{aligned}
\underset{x}{P V} \int \rho\left|\omega^{1 / d}\right| & =\left[\left[\int_{U_{y}} \rho|\xi|^{1 / d} \prod_{i \in I_{x}}\left|x_{i}\right|^{\alpha_{i}+s_{i}}|d x|\right]_{m c}\right]_{s_{x}=0} \\
& =\left[\left[\int_{U_{y}} \rho|\xi|^{1 / d}|\zeta| \prod_{i \in I_{x}}\left|f_{i}\right|^{\alpha_{i}+s_{i}} \prod_{i \in I_{y}}\left|y_{i}\right|^{\alpha_{i}+s_{i}}|d y|\right]_{m c}\right]_{s_{x}=0} \\
& =\left[\left[\int_{U_{y}} \rho|\xi|^{1 / d}|\zeta| \prod_{i \in I_{x}}\left|f_{i}\right|^{\alpha_{i}} \prod_{i \in I_{y}}\left|y_{i}\right|^{\alpha_{i}+s_{i}}|d y|\right]_{m c}\right]_{s_{y}=0} \\
& =P V \int \rho\left|\omega^{1 / d}\right|
\end{aligned}
$$

The first, third and last equality all follow from lemma (3.3).

( 5.9 ) Lemma. Let $\left\{\left(U_{p}, \varphi_{p}, x_{p}\right)\right\}_{p \in P}$ and $\left\{\left(U_{q}, \varphi_{q}, x_{q}\right)\right\}_{q \in Q}$ be two sets of triples as in lemma (5.7). Then

$$
\sum_{p \in P} P V \int \rho \varphi_{p}\left|\omega^{1 / d}\right|=\sum_{q \in Q} P P_{x_{q}} \int \rho \varphi_{q}\left|\omega^{1 / d}\right| .
$$


Proof: For clarity we may assume $P, Q$ (and $X$ ) are disjoint. For every $r$ in $\operatorname{supp}(\rho)$ we can find a pv-chart $x_{r}: U_{r} \rightarrow \mathbb{R}^{m}$ for $(X, \omega)$ such that

1. $x_{r}$ is centered in $r \in U_{r}$ and

2. for all $t \in P \cup Q$ we have $U_{r} \subset U_{t}$ if $r \in U_{t}$ and $U_{r} \cap \operatorname{supp}\left(\varphi_{t}\right)=\emptyset$ if $r \notin U_{t}$.

Then, by the compactness of $\operatorname{supp}(\rho)$ we can take a finite subset $R$ of $\operatorname{supp}(\rho)$ such that $\left\{U_{r}\right\}_{r \in R}$ is a covering of $\operatorname{supp}(\rho)$. Choose a $C^{\infty}$ compact partition of unity $\left\{\varphi_{r}\right\}_{r \in R}$ with respect to this covering. Then

$$
\begin{aligned}
\sum_{p \in P} P V \int \rho \varphi_{p}\left|\omega^{1 / d}\right| & =\sum_{p \in P} \sum_{r \in R \cap U p} P V \int \rho \varphi_{p} \varphi_{r}\left|\omega^{1 / d}\right| \\
& =\sum_{p \in P r \in R \cap U p} \sum_{x_{p}} P V \int \rho \varphi_{p} \varphi_{r}\left|\omega^{1 / d}\right| \\
& =\sum_{r \in R} \sum_{p \in P} P V \int \rho \varphi_{p} \varphi_{r}\left|\omega^{1 / d}\right| \\
& =\sum_{r \in U} P V \int \rho \varphi_{r}\left|\omega^{1 / d}\right| \\
& =\sum_{q \in Q} P V \int \rho \varphi_{x_{q}}\left|\omega^{1 / d}\right|
\end{aligned}
$$

The first and fourth equality follow from lemma (5.7). The second follows from lemma (5.8). The third equality is just changing the summation order. The last equality follows in a similar way as the previous ones.

Lemma (5.6) and lemma (5.9) justify the following definition.

( 5.10 ) Definition. Choose a finite set of triples $\left\{\left(U_{p}, \varphi_{p}, x_{p}\right)\right\}_{p \in P}$ as in the previous lemma, then we define the principal value integral of $\rho\left|\omega^{1 / d}\right|$ on $X$ as follows:

$$
P V \int_{X} \rho\left|\omega^{1 / d}\right|:=\sum_{p \in P} P_{x_{p}} \int \rho \varphi_{p}\left|\omega^{1 / d}\right| .
$$

( 5.11 ) Remark. Let $D$ denote the locus of $\omega$. Sometimes it is convenient to define twisted principal value integrals by multiplying the integrand with a function $\chi: X-D \rightarrow\{1,-1\}$ that is constant on the connected components of 
$X-D$. Formally one extends $\chi$ to a function $\chi: X \rightarrow\{0,1,-1\}$ by defining $\chi(P)=0$ for $P$ in $D$. Then one defines $P V \int_{X} \rho \chi\left|\omega^{1 / d}\right|$ in a similar way as before.

\section{Examples}

( 6.1 ) In general it is difficult to compute principal value integrals since this involves a $C^{\infty}$-partition of unity. In the examples given here we will therefore use the 'globalizing method' to compute some principal value integrals. Let $X$ be a real-analytic compact manifold and $\omega$ a real-analytic differential $m$-form of degree $d$ on $X$ such that $\omega$ has normal crossings on $X$ and $\omega^{1 / d}$ has no integral poles on $X$. Suppose that $h$ is a real-analytic function on $X$ such that the locus of $h$ has normal crossings on $X$ and contains the polar locus of $\omega$. Using a partition of unity one deduces that

$$
P V \int_{X}\left|\omega^{1 / d}\right|=\left[\left[\int_{X}|h|^{s}\left|\omega^{1 / d}\right|\right]_{m c}\right]_{s=0} .
$$

( 6.2 ) Example. Let $a_{0}, a_{1}, a_{\infty} \in \mathbb{Q}-\{0,-1,-2,-3, \ldots\}$ with $a_{0}+a_{1}+$ $a_{\infty}=1$. Let $d$ be a positive integer such that $d a_{x} \in \mathbb{Z}$ for $x \in\{0,1, \infty\}$. Let $N_{0}, N_{1}, N_{\infty} \in\{0,1\}$. Let $\omega$ be the meromorphic differential 1-form of degree $d$ on $\mathbb{P}^{1}(\mathbb{R})$ given on $\mathbb{A}^{1}(\mathbb{R})$ by $X^{d\left(a_{0}-1\right)}(1-X)^{d\left(a_{1}-1\right)}(d X)^{d}$, where $X$ denotes the standard affine coordinate on $\mathbb{A}^{1}(\mathbb{R})$. Define $\chi: \mathbb{P}^{1}(\mathbb{R}) \rightarrow\{-1,0,1\}: X \mapsto$ $\operatorname{sgn}(X(X-1))^{N_{\infty}} \operatorname{sgn}(X)^{N_{1}} \operatorname{sgn}(1-X)^{N_{0}}$. Let $h(X):=\frac{X^{2}(1-X)^{2}}{\left(1+X^{2}\right)^{3}}$. Then

$$
\begin{aligned}
P V \int_{\mathbb{P}(\mathbb{R})} \chi\left|\omega^{1 / d}\right|= & {\left[\left[\int_{\mathbb{P}(\mathbb{R})}|h|^{s} \chi\left|\omega^{1 / d}\right|\right]_{m c}\right]_{s=0} } \\
= & {\left[\left[\int_{-\infty}^{0}|h|^{s} \chi\left|\omega^{1 / d}\right|\right]_{m c}\right]_{s=0} } \\
& +\left[\left[\int_{0}^{1}|h|^{s} \chi\left|\omega^{1 / d}\right|\right]_{m c}\right]_{s=0} \\
& +\left[\left[\int_{1}^{\infty}|h|^{s} \chi\left|\omega^{1 / d}\right|\right]_{m c}\right]_{s=0} \\
= & {\left[\left[\int_{-\infty}^{0} \chi|X|^{a_{0}-1+s}|1-X|^{a_{1}-1+s}|d X|\right]_{m c}\right]_{s=0} } \\
& +\left[\left[\int_{0}^{1} \chi|X|^{a_{0}-1+s}|1-X|^{a_{1}-1+s}|d X|\right]_{m c}\right]_{s=0} \\
& +\left[\left[\left.\int_{1}^{\infty} \chi\right|^{a^{a_{0}-1+s}}|1-X|^{a_{1}-1+s}|d X|\right]_{m c}\right]_{s=0}
\end{aligned}
$$




$$
\begin{aligned}
= & (-1)^{N_{1}}\left[\left[\int_{0}^{1}|Y|^{a_{0}-1+s}|1-Y|^{a_{\infty}-1+s}|d Y|\right]_{m c}\right]_{s=0} \\
& +(-1)^{N_{\infty}}\left[\left[\int_{0}^{1}|Y|^{a_{0}-1+s}|1-Y|^{a_{1}-1+s}|d Y|\right]_{m c}\right]_{s=0} \\
& +(-1)^{N_{0}}\left[\left[\int_{0}^{1}|Y|^{a_{1}-1+s}|1-Y|^{a_{\infty}-1+s}|d Y|\right]_{m c}\right]_{s=0} \\
= & (-1)^{N_{1}} B\left(a_{\infty}, a_{0}\right)+(-1)^{N_{\infty}} B\left(a_{0}, a_{1}\right)+(-1)^{N_{0}} B\left(a_{1}, a_{\infty}\right) \\
= & \frac{1}{\pi} \prod_{x \in\{0,1, \infty\}} \Gamma\left[a_{x}\right] \sum_{x \in\{0,1, \infty\}}\left((-1)^{N_{x}} \sin \left(\pi a_{x}\right)\right) \\
= & \frac{4}{\pi} \prod_{x \in\{0,1, \infty\}=\{x, y, z\}}(-1)^{N_{y} N_{z}} \Gamma\left[a_{x}\right] \cos \left[\frac{\pi}{2}\left(a_{x}+\left|N_{y}-N_{z}\right|\right)\right] .
\end{aligned}
$$

To obtain the first three equations one uses integration by parts and similar techniques as one uses to prove lemma (3.3). In the fourth equation we changed variabels $(X=Y /(Y-1)$ in the first term and $X=1 / Y$ in the third term). The function $B(u, v)$ in $(5)$ denotes the well-known beta function (see e.g. [Bat]). Thus $B(u, v)=\Gamma[u] \Gamma[v] / \Gamma[u+v]$. In (6) we used the reflection formula for the gamma function $([$ Bat, $I, \S 2])$. Finally we get (7) using elementary relations between trigonometric functions.

Let $\{x, y, z\}=\{0,1, \infty\}$. We see there are essentially two different cases. In the first case $N_{x}=N_{y}=N_{z}$ and

$$
P V \int_{\mathbb{P}^{1}(\mathbb{R})} \chi\left|\omega^{1 / d}\right|=(-1)^{N_{x}} \frac{4}{\pi} \prod_{x \in\{0,1, \infty\}} \cos \left[\frac{\pi}{2} a_{x}\right] \Gamma\left[a_{x}\right] .
$$

In the second case $N_{x}=N_{y} \neq N_{z}$ and

$$
P V \int_{\mathbb{P}^{1}(\mathbb{R})} \chi\left|\omega^{1 / d}\right|=(-1)^{N_{x}} \frac{4}{\pi} \sin \left[\frac{\pi}{2} a_{x}\right] \sin \left[\frac{\pi}{2} a_{y}\right] \cos \left[\frac{\pi}{2} a_{z}\right] \prod_{x \in\{0,1, \infty\}} \Gamma\left[a_{x}\right] .
$$

A few important conclusions on the vanishing of these principal value integrals can be drawn. Suppose there exists no $z$ in $\{0,1, \infty\}$ with $a_{z} \in \mathbb{Z}$. Then $P V \int_{\mathbb{P}^{1}(\mathbb{R})} \chi\left|\omega^{1 / d}\right| \neq 0$. Suppose that $a_{z}$ is the only integer in $\left\{a_{0}, a_{1}, a_{\infty}\right\}$. Then $P V \int_{\mathbb{P}^{1}(\mathbb{R})} \chi\left|\omega^{1 / d}\right|=0$ if and only if either $N_{x}=N_{y}$ and $a_{z}$ is odd or $N_{x} \neq N_{y}$ and $a_{z}$ is even.

If the Euler-Poincaré characteristic of $\mathbb{P}^{1}(\mathbb{C})-|\operatorname{div}(\omega)|$ is equal to 0 , there exists (exactly) one element, say $z$, in $\{0,1, \infty\}$ with $a_{z}=1$. If moreover $\chi$ is constant on the connected components of $\mathbb{P}^{1}(\mathbb{R})-|\operatorname{div}(\omega)|$ then $N_{x}=N_{y}$. Thus $P V \int_{\mathbb{P}^{1}(\mathbb{R})} \chi\left|\omega^{1 / d}\right|=0$. This result also follows from the more general result on the vanishing of principal value integrals mentioned in the introduction (1.1). 
( 6.3 ) Example. Let $(x ; y ; z)$ be projective coordinates on $\mathbb{P}^{2}(\mathbb{R})$. Let $U_{y}:=\{(x ; y ; z) \mid y \neq 0\}$ and $U_{z}:=\{(x ; y ; z) \mid z \neq 0\}$ and use affine coordinates $\left(X_{y}, Z_{y}\right):=(x / y, z / y)$ on $U_{y}$ and $\left(X_{z}, Y_{z}\right):=(x / z, y / z)$ on $U_{z}$. Let $\omega$ be the meromorphic differential 2-form of degree 2 on $\mathbb{P}^{2}(\mathbb{R})$ given on $U_{y}$ by

$$
\left(Z_{y}-X_{y}^{2}\right)^{-3}\left(d X_{y} d Z_{y}\right)^{2} .
$$

Then on $U_{z}$ we have

$$
\omega=\left(Y_{z}-X_{z}^{2}\right)^{-3}\left(d X_{z} d Y_{z}\right)^{2} .
$$

Let $h=\frac{y z-x^{2}}{x^{2}+y^{2}+z^{2}}$ on $\mathbb{P}^{2}(\mathbb{R})$. Then

$$
\begin{aligned}
P V \int_{\mathbb{P}^{2}(\mathbb{R})}\left|\omega^{1 / 2}\right|= & {\left[\left[\int_{\mathbb{P}^{2}(\mathbb{R})}|h|^{s}\left|\omega^{1 / 2}\right|\right]_{m c}\right]_{s=0} } \\
= & {\left[\left[\int_{\left|Y_{z}\right|<1}\left|Y_{z}-X_{z}^{2}\right|^{-3 / 2+s}\left|X_{z}^{2}+Y_{z}^{2}+1\right|^{-s}\left|d X_{z} d Y_{z}\right|\right]_{m c}\right]_{s=0} } \\
& +\left[\left[\int_{\left|Z_{y}\right|<1}\left|Z_{y}-X_{y}^{2}\right|^{-3 / 2+s}\left|X_{y}^{2}+1+Z_{y}^{2}\right|^{-s}\left|d X_{y} d Z_{y}\right|\right]_{m c}\right]_{s=0} \\
= & 2\left[\left[\int_{\left|Y_{z}\right|<1}\left|Y_{z}-X_{z}^{2}\right|^{-3 / 2+s}\left|X_{z}^{2}+Y_{z}^{2}+1\right|^{-s}\left|d X_{z} d Y_{z}\right|\right]_{m c}\right]_{s=0}
\end{aligned}
$$

Now

$$
\begin{gathered}
\int_{\left|Y_{z}\right|<1}\left|Y_{z}-X_{z}^{2}\right|^{-3 / 2+s}\left|X_{z}^{2}+Y_{z}^{2}+1\right|^{-s}\left|d X_{z} d Y_{z}\right| \\
=\int_{X=-\infty}^{\infty} \int_{T=-X^{2}-1}^{-X^{2}+1}|T|^{-3 / 2+s}\left|X^{2}+\left(T+X^{2}\right)^{2}+1\right|^{-s} d T d X \\
=\frac{1}{s-1 / 2} \int_{X=-\infty}^{\infty}\left(X^{2}+2\right)^{-s}\left(\operatorname{sgn}\left(1-X^{2}\right)\left|1-X^{2}\right|^{s-1 / 2}+\left|1+X^{2}\right|^{s-1 / 2}\right) d X \\
+\frac{2 s}{s-1 / 2} \int_{X=-\infty}^{\infty} \int_{T=-X^{2}-1}^{-X^{2}+1} \operatorname{sgn}(T)|T|^{s-1 / 2}\left|X^{2}+\left(T+X^{2}\right)^{2}+1\right|^{-s-1}\left(T+X^{2}\right) d T d X .
\end{gathered}
$$

The integral in the first expression is analytic for $\operatorname{Re}(s)>1 / 2$. The first integral in the last expression is analytic for $\operatorname{Re}(s)>-1 / 2$ and the second integral in the last expression is analytic for $\operatorname{Re}(s)>-1 / 6$. Thus

$$
\begin{gathered}
P V \int_{\mathbb{P}^{2}(\mathbb{R})}\left|\omega^{1 / 2}\right|= \\
2\left[\left[\int_{X=-\infty}^{\infty} \frac{\left(X^{2}+2\right)^{-s}}{s-1 / 2}\left(\operatorname{sgn}\left(1-X^{2}\right)\left|1-X^{2}\right|^{s-1 / 2}+\left|1+X^{2}\right|^{s-1 / 2}\right) d X\right]_{m c}\right]_{s=0} \\
=-\int_{X=-\infty}^{\infty}\left(\operatorname{sgn}\left(1-X^{2}\right)\left|1-X^{2}\right|^{-1 / 2}+\left|1+X^{2}\right|^{-1 / 2}\right) d X \\
=-4 \pi .
\end{gathered}
$$




\section{Generalization of the definition of principal value integrals on manifolds}

( 7.1 ) Data. In this section we use the same data as in section 5. Thus $X$ is a $m$-dimensional real-analytic manifold, $\omega$ is a real meromorphic differential $m$-form of degree $d$ on $X$ and $\rho: X \rightarrow \mathbb{R}$ is a $C^{\infty}$-function with compact support.

( 7.2 ) Proposition. Let $X, Y$ be two real-analytic manifolds of dimension $m$. Let $\pi: Y \rightarrow X$ be a real-analytic map. Let $(P, \omega)$ be a real-meromorphic differential $m$-form of degree $d$ on $X$ such that $Y-\pi^{-1}(P)$ is dense in $Y$. Then the real-analytic differential $m$-form $\pi^{*}(\omega)$ of degree $d$ on $Y-\pi^{-1}(P)$ has a unique real-meromorphic extension to $Y$, denoted by $\pi^{*}(P, \omega)$.

Proof: This follows directly from lemma (2.9).

( 7.3 ) Remark. Suppose that $Y$ is connected and that there exists a realanalytic subset $D$ of codimension at least 1 in $X$ such that $\pi: Y-\pi^{-1}(D) \rightarrow$ $X-D$ is a real-analytic isomorphism. Then $Y-\pi^{-1}(P)$ is dense in $Y$.

( 7.4 ) Remark. Suppose we have three real-analytic manifolds $X, Y, Z$ of dimension $m$, a real-meromorphic differential $m$-form $(P, \omega)$ of degree $d$ on $X$ and real-analytic maps $\pi_{1}: Y \rightarrow X, \pi_{2}: Z \rightarrow Y$ such that $Y-\pi_{1}^{-1}(P)$ is dense in $Y$ and $Z-\left(\pi_{1} \circ \pi_{2}\right)^{-1}(P)$ is dense in $Z$. Then $\pi_{2}^{*}\left(\pi_{1}^{*}(P, \omega)\right)=\left(\pi_{1} \circ \pi_{2}\right)^{*}(P, \omega)$.

( 7.5 ) Remark. Denote the standard coordinates on $\mathbb{A}^{2}(\mathbb{R})$, resp. $\mathbb{P}^{1}(\mathbb{R})$, by $\left(x_{1}, x_{2}\right)$, resp. $\left(y_{1}, y_{2}\right)$. Let $X=\mathbb{A}^{2}(\mathbb{R})$ and let $Y$ be the Zariski closed subset of $\mathbb{P}^{1}(\mathbb{R}) \times \mathbb{A}^{2}(\mathbb{R})$ given by the equation $y_{1} x_{2}=y_{2} x_{1}$. Let $\pi: Y \rightarrow X$ be the projection which sends $\left(y_{1}, y_{2}, x_{1}, x_{2}\right)$ to $\left(x_{1}, x_{2}\right)$. Let $P$ be the $x_{1}$-axis on $X$ and $\omega=\frac{d x_{1} \wedge d x_{2}}{x_{2}}$ on $X-P$. Then the polar locus of $\pi^{*}(P, \omega)$ is given by $y_{2}=0$ on $Y$ and is strictly included in $\pi^{-1}(P)$.

( 7.6 ) Definition. Suppose first that $X$ is connected. Let $U$ be the complement in $X$ of the locus of $\omega$. We say that $\pi: Y \rightarrow X$ is an embedded resolution of $(X, \omega, \rho)$ if

1. $Y$ is a real-analytic $m$-dimensional connected manifold,

2. $\pi$ is a proper real-analytic morphism,

3. $\pi: \pi^{-1}(U) \rightarrow U$ is a real-analytic isomorphism and 
4. $\pi^{*}(\omega)$ has normal crossings in $\operatorname{supp}\left(\pi^{*}(\rho)\right)$.

If $X$ has connected components $X_{\alpha}$, we say that $\pi: Y \rightarrow X$ is an embedded resolution of $(X, \omega, \rho)$ if the connected components of $Y$ are in 1-1 correspondence to those of $X$ (say $X_{\alpha}$ corresponds to $Y_{\alpha}$ ), $\pi\left(Y_{\alpha}\right) \subset X_{\alpha}$ and $\pi \mid Y_{\alpha}: Y_{\alpha} \rightarrow X_{\alpha}$ is an embedded resolution of $\left(X_{\alpha}, \omega\left|X_{\alpha}, \rho\right| X_{\alpha}\right)$ for each $\alpha$.

( 7.7 ) Definition. Let $x$ and $h$ be as in lemma (2.13). Then we call $h$ a local pv-function for $(x, \omega)$.

( 7.8) Definition. Let $h$ be a real-meromorphic function on an open subset $V$ of $X$. We say that the couple $(h, \omega)$ has normal crossings in a point $p$ of $V$ if there exist an admissible chart $x: U \rightarrow \mathbb{R}^{m}$ for $(X, \omega)$ with data $(\xi, \beta)$, centered in $p$, integers $\gamma_{i}$ and a nowhere vanishing real-analytic function $\eta$ on $U$ such that $U \subset V$ and

$$
h=\eta \prod_{i=1}^{m} x_{i}^{\gamma_{i}}
$$

on $U$. Such a chart is called an admissible chart for $(X, h, \omega)$ with data $(\eta ; \gamma ; \xi ; \beta)$. If the $\beta_{i} / d \notin \mathbb{Z}-\mathbb{N}$ and the $\gamma_{i} \in \mathbb{N}$ then we call it a pv-chart for $(X, h, \omega)$ with data $(\eta ; \gamma ; \xi ; \beta)$. We say that $(h, \omega)$ has normal crossings in a subset $K$ of $V$ if it has normal crossings in every point of $K$.

( 7.9 ) Definition. Let $Y$ be a real-analytic $m$-dimensional manifold. Let $\pi: Y \rightarrow X$ be an embedded resolution of $(X, \omega, \rho)$ (see (7.6)). We say that $\pi$ is a good resolution of $(X, \omega, \rho)$ if for every point $p$ of $\operatorname{supp}(\rho)$ there exists a chart $x: U \rightarrow \mathbb{R}^{m}$ on $X$ centered in $p$ and a local pv-function $h$ for $(x, \omega)$ such that $\left(\pi^{*}(h), \pi^{*}(\omega)\right)$ has normal crossings in $\operatorname{supp}(\rho) \cap \pi^{-1}(U)$. We say that $\pi$ is an embedded pv-resolution of $(X, \omega, \rho)$ if

1. $\pi^{*}\left(\omega^{1 / d}\right)$ has no integral poles in $\operatorname{supp}\left(\pi^{*}(\rho)\right)$ and

2. $\pi$ is a good resolution of $(X, \omega, \rho)$.

( 7.10 ) Remark. Note that only the first condition is needed to define $P V \int_{Y} \pi^{*}(\rho)\left|\pi^{*}(\omega)^{1 / d}\right|$. We need the second one to prove that $P V \int_{Y} \pi^{*}(\rho)\left|\pi^{*}(\omega)^{1 / d}\right|$ is independent of the chosen resolution. Remark that Hironaka's theorem on resolutions of singularities (see [Hi1]) implies that there always exists a good resolution for $(X, \omega, \rho)$. 
( 7.11 ) Remark. We give an example in which the first condition is satisfied but the second is not. Let

$$
\pi: \mathbb{P}^{2}(\mathbb{R}) \rightarrow \mathbb{P}^{2}(\mathbb{R}):\left(x_{0} ; x_{1} ; x_{2}\right) \rightarrow\left(x_{0}^{3}, x_{1}\left(x_{1}^{2}+x_{2}^{2}\right), x_{2}\left(x_{1}^{2}+x_{2}^{2}\right)\right) .
$$

Let $U_{0}$ be the affine open subset of $\mathbb{P}^{2}(\mathbb{R})$ given by $x_{0} \neq 0$. Let $X=\left(X_{1}, X_{2}\right)=$ $\left(x_{1} / x_{0}, x_{2} / x_{0}\right)$ be a chart on $U_{0}$ and let $\omega$ be given on $U_{0}$ by $\frac{X_{1}}{X_{2}^{5}}\left(d X_{1} \wedge d X_{2}\right)^{2}$. Then $\pi^{*}(\omega)=9 \frac{X_{1}}{X_{2}^{5}}\left(d X_{1} \wedge d X_{2}\right)^{2}$ on $U_{0}$. Suppose that $\operatorname{supp}(\rho) \subset U_{0}$. Then $\pi$ is a resolution for $(X, \omega, \rho)$ which satisfies the first condition. Now $X_{2}^{5}$ is a local pv-function for $(X, \omega)$ and $\pi^{*}\left(X_{2}^{5}\right)=X_{2}^{5}\left(X_{1}^{2}+X_{2}^{2}\right)^{5}$ on $U_{0}$. Thus the second condition is not satisfied.

( 7.12 ) Theorem. Let $\left\{x_{p}: X \rightarrow \mathbb{R}^{m}\right\}_{p \in P}$ be a finite set of charts on $X$ such that the $U_{p}$ cover $\operatorname{supp}(\rho)$ and on each $U_{p}$ we have $\omega=\frac{g_{p}}{h_{p}} d x_{p}$ where $g_{p}$ and $h_{p}$ are locally relatively prime real-analytic functions on $U_{p}$ (see definition (2.12)). Choose a $C^{\infty}$ compact partition of unity $\left\{\varphi_{p}: X \rightarrow \mathbb{R}\right\}_{p \in P}$ with respect to the covering $\left\{U_{p}\right\}_{p \in P}$ of $\operatorname{supp}(\rho)$. Let $\pi: Y \rightarrow X$ be an embedded pv-resolution of $(X, \omega, \rho)$. Then

$$
\left[\left[\int_{U_{p}} \rho \varphi_{p}\left|h_{p}\right|^{s}\left|\omega^{1 / d}\right|\right]_{m c}\right]_{s=0}
$$

exists for each $p$ in $P$ and

$$
P V \int_{Y} \pi^{*}(\rho)\left|\pi^{*}(\omega)^{1 / d}\right|=\sum_{p \in P}\left[\left[\int_{U_{p}} \rho \varphi_{p}\left|h_{p}\right|^{s}\left|\omega^{1 / d}\right|\right]_{m c}\right]_{s=0}
$$

Proof: We may suppose that $X$ and $Y$ are connected. We have $\operatorname{supp}\left(\pi^{*}\left(\rho \varphi_{p}\right)\right)$ is compact since it is contained in $\pi^{-1}\left(\operatorname{supp}\left(\rho \varphi_{p}\right)\right)$, which is compact by the properness of $\pi$. For every $p \in P$ choose a finite set $Q_{p}$ and for all $q \in Q_{p}$ a pv-chart $y_{q}: V_{q} \rightarrow \mathbb{R}^{m}$ for $\left(Y, \pi^{*}\left(h_{p}\right), \pi^{*}(\omega)\right)$ with data $(\eta ; \gamma ; \xi ; \beta)$, centered in a point of $V_{q}$, and such that $\left\{V_{q}\right\}_{q \in Q_{p}}$ is a covering of $\operatorname{supp}\left(\pi^{*}\left(\rho \varphi_{p}\right)\right)$ and $V_{q} \subset \pi^{-1}\left(U_{p}\right)$. Choose a $C^{\infty}$ compact partition of unity $\left\{\psi_{q}\right\}_{q \in Q_{p}}$ with respect to this covering.Fix $p \in P, q \in Q_{p}$ and let $U=U_{p}, x=x_{p}, \varphi=\varphi_{p}, h=h_{p}$, $y=y_{q}, V=V_{q}$ and $\psi=\psi_{q}$. On $V$ we have :

$$
\pi^{*}(\omega)=\xi \prod_{i=1}^{m} y_{i}^{\beta_{i}} d y
$$

and

$$
\pi^{*}(h)=\eta \prod_{i=1}^{m} y_{i}^{\gamma_{i}}
$$


with $\gamma_{i}>0$ if $\beta_{i}<0$. Then

$$
\begin{aligned}
\underset{y}{P V} \int \pi^{*}(\rho \varphi) \psi\left|\pi^{*}(\omega)^{1 / d}\right| & =\left[\left[\int_{V} \pi^{*}(\rho \varphi) \psi|\xi|^{1 / d} \prod_{i=1}^{m}\left|y_{i}\right|^{\beta_{i} / d+s}|d y|\right]_{m c}\right]_{s=0} \\
& =\left[\left[\int_{V} \pi^{*}(\rho \varphi) \psi|\xi|^{1 / d}|\eta|^{s} \prod_{i=1}^{m}\left|y_{i}\right|^{\beta_{i} / d+\gamma_{i} s}|d y|\right]_{m c}\right]_{s=0} \\
& =\left[\left[\int_{V} \pi^{*}(\rho \varphi) \psi\left|\pi^{*}(h)\right|^{s}\left|\pi^{*}(\omega)^{1 / d}\right|\right]_{m c}\right]_{s=0}
\end{aligned}
$$

Here the second equality follows from lemma (3.3). Then for $p \in P$ we have:

$$
\begin{aligned}
\sum_{q \in Q_{p}} \underset{y_{q}}{P V} \int \pi^{*}\left(\rho \varphi_{p}\right) \psi_{q}\left|\pi^{*}(\omega)^{1 / d}\right| & =\sum_{q \in Q_{p}}\left[\left[\int_{V_{q}} \pi^{*}\left(\rho \varphi_{p}\right) \psi_{q}\left|\pi^{*}\left(h_{p}\right)\right|^{s}\left|\pi^{*}(\omega)^{1 / d}\right|\right]_{m c}\right]_{s=0} \\
& =\left[\left[\int_{\pi^{-1}\left(U_{p}\right)} \pi^{*}\left(\rho \varphi_{p}\right)\left|\pi^{*}\left(h_{p}\right)\right|^{s}\left|\pi^{*}(\omega)^{1 / d}\right|\right]_{m c}\right]_{s=0} \\
& =\left[\left[\int_{U_{p}} \rho \varphi_{p}\left|h_{p}\right|^{s}\left|\omega^{1 / d}\right|\right]_{m c}\right]_{s=0}
\end{aligned}
$$

Here the last equality follows since $\pi: Y-\pi^{-1}(D) \rightarrow X-D$ is a real-analytic isomorphism, where $D$ denotes the locus of $\omega$.

Then one easily checks that $\left\{V_{q} \mid q \in \cup_{p \in P} Q_{p}\right\}$ is a finite covering of $\operatorname{supp}\left(\pi^{*}(\rho)\right)$ and $\left\{\pi^{*}\left(\varphi_{p}\right) \psi_{q} \mid p \in P, q \in Q_{p}\right\}$ is a $C^{\infty}$ compact partition of unity with respect to this covering. Thus

$$
\begin{aligned}
P V \int_{Y} \pi^{*}(\rho)\left|\pi^{*}(\omega)^{1 / d}\right| & =\sum_{p \in P} \sum_{q \in Q_{p}} P V \int \pi^{*}\left(\rho \varphi_{p}\right) \psi_{q}\left|\pi^{*}(\omega)^{1 / d}\right| \\
& =\sum_{p \in P}\left[\left[\int_{U_{p}} \rho \varphi_{p}\left|h_{p}\right|^{s}\left|\omega^{1 / d}\right|\right]_{m c}\right]_{s=0}
\end{aligned}
$$

( 7.13 ) Corollary. Let $\pi_{i}: Y_{i} \rightarrow X, i=1,2$, be two embedded pv-resolutions of $(X, \omega, \rho)$. Then

$$
P V \int_{Y_{1}} \pi_{1}^{*}(\rho)\left|\pi_{1}^{*}(\omega)^{1 / d}\right|=P V \int_{Y_{2}} \pi_{2}^{*}(\rho)\left|\pi_{2}^{*}(\omega)^{1 / d}\right|
$$


Proof: This follows directly from theorem (7.12).

( 7.14 ) Definition. Assume that there exists an embedded pv-resolution $\pi: Y \rightarrow X$ of $(X, \omega, \rho)$. Then we say that the principal value integral of $\rho\left|\omega^{1 / d}\right|$ on $X$ exists and define it as follows:

$$
P V \int_{X} \rho\left|\omega^{1 / d}\right|:=P V \int_{Y} \pi^{*}(\rho)\left|\pi^{*}(\omega)^{1 / d}\right|
$$

( 7.15 ) Definition. Let $Y$ be a real-analytic manifold of dimension $m$ and let $\pi: Y \rightarrow X$ be a proper real-analytic map. Let $D$ denote the lodus of $\omega$. We say that $\pi$ is a pv-map for $(X, \omega)$ if

1. $\pi \mid\left(Y-\pi^{-1}(D)\right)$ is a real-analytic isomorphism onto $X-D$,

2. $Y-\pi^{-1}(D)$ is dense in $Y$ and

3. if $V$ is an open in $X$ and $h$ is a pv-function for $(V, \omega)$ then $\pi^{*}(h)$ is a pv-function for $\left(\pi^{-1}(V), \pi^{*}(\omega)\right)$.

( 7.16 ) Corollary. Let $\pi: Y \rightarrow X$ be a pv-map for $(X, \omega)$. Suppose that $P V \int_{Y} \pi^{*}(\rho)\left|\pi^{*}(\omega)^{1 / d}\right|$ exists. Then $P V \int_{X} \rho\left|\omega^{1 / d}\right|$ exists and

$$
P V \int_{X} \rho\left|\omega^{1 / d}\right|=P V \int_{Y} \pi^{*}(\rho)\left|\pi^{*}(\omega)^{1 / d}\right|
$$

Proof: Choose a pv-resolution $\varphi: Z \rightarrow Y$ for $\left(Y, \pi^{*}(\omega), \pi^{*}(\rho)\right)$. Then $\varphi \circ \pi$ is a pv-resolution for $(X, \omega, \rho)$ since $\pi$ is a pv-map for $(X, \omega)$. This proves the corollary.

( 7.17 ) Remark. Similar to the normal crossings case, one can also define the twisted principal value integral $P V \int_{X} \rho \chi\left|\omega^{1 / d}\right|$ by multiplying the integrand with a function $\chi: X \rightarrow 0,1,-1$ where $\chi(P)=0$ if and only if $P \in D$ and where $\chi$ is constant on the connected components of $X-D$. Also corrolary (7.16) remains valid. 


\section{Compactifications}

Let $Y$ be a real-analytic manifold of dimension $m$ and $\omega$ a meromorphic differential $m$-form on $Y$ of degree $d$. Let $i: Y \rightarrow X$ be a compactification of $Y$, i.e. $i$ is a real-analytic embedding of real-analytic manifolds of dimension $m, i(Y)$ is dense in $X$ and $X$ is compact. Then there exists a unique meromorphic differential $m$-form $\hat{\omega}$ of degree $d$ on $X$ such that $\omega=i^{*}(\hat{\omega})$ because of lemma $(2.8)$. Suppose that we are given two compactifications $i_{1}: Y \rightarrow X_{1}, i_{2}: Y \rightarrow X_{2}$ and let $\omega_{1}, \omega_{2}$ be the extensions of $\omega$ with respect to $i_{1}$ and $i_{2}$. Assume that the principal value integrals $P V \int_{X_{i}}\left|\omega_{i}^{1 / d}\right|$ exist. Then one could ask if

$$
P V \int_{X_{1}}\left|\omega_{1}^{1 / d}\right|=P V \int_{X_{2}}\left|\omega_{2}^{1 / d}\right| ?
$$

We show that the answer is negative. For this purpose let $C$ be the curve on $\mathbb{P}^{2}(\mathbb{R})$ given in standard affine coordinates $(X, Y)$ by $Y-X^{2}=0$ and let $L$ be the line tangent to the point at infinity of $C$. Let $L_{1}, L_{2}$ be two different lines on $\mathbb{P}^{2}(\mathbb{R})$. Denote $\mathbb{P}^{2}(\mathbb{R})-(C \cup L)$ by $U$ and $\mathbb{P}^{2}(\mathbb{R})-\left(L_{1} \cup L_{2}\right)$ by $V$. Denote the inclusion $U \subset \mathbb{P}^{2}(\mathbb{R})$ by $j_{1}$ and the inclusion $V \subset \mathbb{P}^{2}(\mathbb{R})$ by $j_{2}$. Let $\omega$ be the real-analytic differential 2-form of degree 2 on $U$ given by $\left(Y-X^{2}\right)^{-3}(d X d Y)^{2}$ (see example (6.3)) and denote the extension of $\omega$ with respect to $j_{1}$ by $\omega_{1}$. Then there exists an isomorphism $f: U \rightarrow V$ such that $\operatorname{div}\left(\omega_{2}\right)=-3 L_{1}-3 L_{2}$, where $\omega_{2}$ denotes the extension of $\left(f^{-1}\right)^{*}(\omega)$ with respect to $j_{2}$. This isomorphism can be obtained by constructing a row of blowings-up and blowings-down starting and ending in $\mathbb{P}^{2}(\mathbb{R})$, as pointed out by W.Veys. A calculation similar to those of section 6 shows that $P V \int_{\mathbb{P}^{2}(\mathbb{R})}\left|\omega_{2}^{1 / d}\right|=0$. This is also an application of a Theorem which gives a sufficient condition for the vanishing of principal value integrals on algebraic varieties in terms of cohomology, see the introduction or [D-J, Main Theorem 1.1.4]. From example (6.3) we know however that $P V \int_{\mathbb{P}^{2}(\mathbb{R})}\left|\omega_{1}^{1 / d}\right| \neq 0$.

\section{Limits of principal value integrals}

( 9.1 ) Data. Let $X$ be a $m$-dimensional real-analytic manifold. Let $\omega$ be a real meromorphic differential $m$-form of degree $d$ on $X$. Let $\rho: X \rightarrow \mathbb{R}$ be a $C^{\infty}$-function with compact support and let $g$ be a real meromorphic function on $X$.

( 9.2$)$ Proposition. Assume that there exists an embedded pv-resolution $\pi: Y \rightarrow X$ of $(X, \omega, \rho)$ which is also an embedded pv-resolution of $\left(X, g \omega^{n}, \rho\right)$ 
for $n$ big enough. Then

$$
\lim _{n \rightarrow \infty} P V \int_{X} \rho\left|\left(g \omega^{n}\right)^{1 / n d}\right|=P V \int_{X} \rho\left|\omega^{1 / d}\right| .
$$

Proof: $\quad$ Since $\pi$ is an embedded pv-resolution of $(X, \omega, \rho)$ and of $\left(X, g \omega^{n}, \rho\right)$ for $n$ big enough, we may as well assume that $\omega$ and $g \omega^{n}$ have normal crossings on $\operatorname{supp}(\rho)$ and that $\omega^{1 / d}$ and $\left(g \omega^{n}\right)^{1 / n d}$ have no integral poles on $\operatorname{supp}(\rho)$ for $n>>0$. Since this holds for $n$ arbitrary large, $(g, \omega)$ must have normal crossings on $\operatorname{supp}(\rho)$. Thus we can find a finite set of triples $\left\{\left(U_{p}, \varphi_{p}, x_{p}\right)\right\}$ such that $\left\{U_{p}\right\}$ is a covering of $\operatorname{supp}(\rho),\left\{\varphi_{p}\right\}$ is a $C^{\infty}$-partition of unity with respect to this covering and $\left(U_{p}, x_{p}\right)$ is an admissible chart for $(X, g, \omega)$. Hence it suffices to prove, for each $p$, that

$$
\lim _{n \rightarrow \infty} \underset{x_{p}}{P V} \int \rho \varphi_{p}\left|\left(g \omega^{n}\right)^{1 / n d}\right|=P_{x_{p}} \int \rho \varphi_{p}\left|\omega^{1 / d}\right| .
$$

Fix $p$ and let $(\eta ; \gamma ; \xi ; \beta)$ be the data of the chart $x_{p}$. Then we have

$$
\begin{aligned}
& \lim _{n \rightarrow \infty} \underset{x_{p}}{P V} \int \rho \varphi_{p}\left|\left(g \omega^{n}\right)^{1 / n d}\right| \\
& =\lim _{n \rightarrow \infty}\left[\left[\int_{U_{p}} \rho \varphi_{p}|\eta|^{1 / n d}|\xi|^{1 / d}|x|^{\frac{\gamma}{n d}+\frac{\beta}{d}+s}|d x|\right]_{m c}\right]_{s=0} \\
& =\left[\left[\int_{U_{p}} \rho \varphi_{p}|\eta|^{t}|\xi|^{1 / d}|x|^{t+\frac{\beta}{d}+s}|d x|\right]_{m c}\right]_{(s, t)=0} \\
& =\left[\left[\int_{U_{p}} \rho \varphi_{p}|\eta|^{t}|\xi|^{1 / d}|x|^{\frac{\beta}{d}+s}|d x|\right]_{m c}\right]_{(s, t)=0} \\
& =P V \int \rho \varphi_{p}\left|\omega^{1 / d}\right| .
\end{aligned}
$$

Here we made use of lemma (3.3).

( 9.3 ) Remark. Let $X=\mathbb{P}^{2}(\mathbb{R})$ and use affine coordinates $(x, y)$ on $X$. Let $\omega=x^{\beta_{1}} y^{\beta_{2}}(x+y+1)^{\beta_{3}}(d x d y)^{d}, d \geq 2$, where $\beta_{1}+\beta_{2}=-2 d$ and $\beta_{1} / d, \beta_{2} / d, \beta_{3} / d \notin \mathbb{Z}$. Let $g=x-y$. Then the identity map $i d_{X}$ on $X$ is an embedded pv-resolution of $(X, \omega, 1)$. On the other hand let $\pi$ be the blowing up of $X$ with center equal to the origin $O$. Then $\pi$ is an embedded pv-resolution of $\left(X, g \omega^{n}, 1\right)$ for each $n \in \mathbb{N}, n>>0$. The coefficient of the exceptional curve in the divisor $\operatorname{div}\left(\pi^{*}\left(g \omega^{n}\right)^{1 / n d}\right)$ is equal to $-1+\frac{1}{n d}$. Note that in this example $\pi$ is not an embedded pv-resolution of $(X, \omega, 1)$ and $i d_{X}$ is not an embedded pv-resolution of $\left(X, g \omega^{n}, 1\right)$. 


\section{References}

[A-V-G] V.ARNOLD, A.VARCHENKO and S.GOUSSEIN-ZADE, Singularités des applications différentiables Tome 2, Editions Mir, Moscou, 1986.

[At] M.F.ATIYAH, Resolution of singularities and division of distributions, Comm. Pure Appl. Math. 23 (1970),145-150.

[Ba1] D.BARLET, Développement asymptotique des fonctions obtenus par intégration sur les fibres, Inv.Math. 68(1982), 129-174.

[Ba2] D.BARLET, Contribution effective de la monodromie aux développements asymptotiques, Ann.Sci.Ec.Norm.Sup. 17(1984),293-315.

[Ba3] D.BARLET, Contribution effective dans le réel, Compositio Math. 56 (1985), 351-359.

[Ba4] D.BARLET, Le calcul de la forme hermitienne canonique pour un polynôme homogène à singularité isolée, Journal fur die reine und angewandte Mathematik 362(1985), 179-196.

[Ba-Ma] D.BARLET and A.MARDHY, Un critère topologique d'existence de pôles pour le prolongement méromorphe de $\int_{A} f^{s} \square$, Ann.Fourier 43 (1993), 743-750 et erratum 44, 629-630.

[Bat] H.BATEMAN, Higher transcendental functions Volume I, McGraw-Hill Book Company, 1953.

[Be] I.N.BERNSTEIN, The analytic continuation of generalized functions with respect to a parameter, Funct. Anal. Appl. 6 (1972),273285 .

[Be-Ge] I.N.BERNSTEIN and S.I.GELFAND, Meromorphic property of the function $P^{\lambda}$, Funct. Anal. Appl. 3 (1969), 68-69.

[Br] G.E.BREDON, Topology and Geometry, Graduate Texts in Mathematics 139, Springer Verlag, 1993.

[Den1] J.DENEF, Multiplicity of the poles of the Poincaré series of a p-adic subanalytic set, Séminaire de Théorie des Nombres de Bordeaux,1987-1988,Exposé n.43. 
[Den2] J.DENEF, Report on Igusa's local zeta function, Séminaire Bourbaki, 741(1990-91).

[Den3] J.DENEF, Degree of local zeta functions and monodromy, Compos.Math. 89(1993), 207-216.

[D-J] J.DENEF and P.JACOBS, On the vanishing of principal value integrals, Comptes Rendus de l'Acad. des Sciences de Paris, 326(1998), Série I, 1041-1046.

[De-La-Sa] J.DENEF,A.LAEREMANS and P.SARGOS, On the Largest Nontrivial Pole of the Distribution $|f|^{s}$, RIMS Kô kyû roku $\mathbf{9 9 9}(1997), 1-9$.

[De-Sa] J.DENEF and P.SARGOS, Polyèdre de Newton et distribution $f_{+}^{s}$. I, J. Analyse Math. 53(1989),201-218; Polyèdre de Newton et distribution $f_{+}^{s}$. II, Math. Ann. 293(992),193-211.

[Gr-Ha] P.GRIFFITHS AND J.HARRIS, Principles of Algebraic Geometry, John Wiley and Sons,Inc.,1978.

[Gu-Ro] R.C.GUNNING AND H.ROSSI, Analytic functions of several complex variables, Prentice Hall,Inc.,1965.

[Hi1] H.HIRONAKA, Resolution of singularities of an algebraic variety over a field of characteristic zero, Ann. Math. 79(1964),109-326.

[Ho] L.HORMANDER, The Analysis of Linear Partial Differential Operators, Springer Verlag, Berlin Heidelberg, 1983.

[I1] J.IGUSA, Complex powers and asymptotic expansions I, J. reine angew. Math. 268/269(1974),110-130; ; II, ibid $\mathbf{2 7 8 / 2 7 9}(1975), 307-321$.

[I2] J.IGUSA, Lectures on forms of higher degree, Tata Inst.Fund.Research, Bombay, 1978.

[J] Ph.JACOBS, Principal value integrals, cohomology and Igusa's zeta functions, Ph.D. Thesis, Catholic Univ. of Leuven, Belgium, 1998, http://www.wis.kuleuven.ac.be/wis/algebra/Jacobs/geheel.dvi

[J2] Ph.JACOBS, Distributions $|f|^{s}$, oscillating integrals and real principal value integrals, in preparation. 
[Je] A.JEDDI, Singularités isolées dans le réel, Ann. Fourier 41 (1991), $87-116$.

[Lae] A.LAEREMANS, The Distribution $|f|^{s}$, Topological Zeta Functions and Newton Polyhedra, Ph.D. thesis, KULeuven, 1997.

[La1] S.LANG, Analysis II, Addison-Wesley Publishing Company, 1969.

[La2] S.LANG, Complex Analysis, Addison-Wesley Publishing Company, 1977 .

[L1] R.P.LANGLANDS, Orbital integrals on forms of Sl(3),I, Amer.J.Math. 105(1983),465-506.

[L2] R.P.LANGLANDS, Remarks on Igusa theory and real orbital integrals, published in The Zeta Functions of Picard Modular Surfaces, Les Publications CRM, Montréal(1992), distributed by AMS.

[LS1] R.P.LANGLANDS AND D.SHELSTAD, On principal values on $p$ adic manifolds, Springer Lecture Notes in Math., 1041(1984).

[LS2] R.P.LANGLANDS AND D.SHELSTAD, Orbital integrals on forms of Sl(3),II, Can.J.Math. 41(1989),480-507.

[Lo1] F.LOESER, Fonctions d'Igusa p-adiques et polynômes de Bernstein, Amer.J.Math. 110(1988),1-22.

[Lo2] F.LOESER, Fonctions d'Igusa p-adiques, polynômes de Bernstein, et polyèdres de Newton, J. reine angew. Math. 412(1990),75-96.

[Mal] B.MALGRANGE, Intégrales asymptotiques et monodromie, Ann.Scient.Ec.Norm.Sup. 7(1974), 405-430.

[Ve] W.VEYS, Poles of Igusa's local zeta function and monodromy, Bull. Soc. France 121 (1993),545-598.

[Ve2] W.VEYS, Embedded resoultion of singularities and Igusa's local zeta function, Academiae Analecta, to appear.

[We] A.WEIL, Sur la formule de Siegel dans la théorie des groupes classiques, Acta Math. 113(1965),1-87. 
Philippe Jacobs

University of Leuven

Department of Mathematics

Celestijnenlaan 200 B

B-3001 Leuven

Belgium

e-mail:Philippe.Jacobs@wis.kuleuven.ac.be 